\title{
Theory of noise in optical transmission systems repeated by two-mode phase-sensitive optical parametric amplifiers
}

This paper was downloaded from TechRxiv (https://www.techrxiv.org).

\section{LICENSE}

CC BY 4.0

SUBMISSION DATE / POSTED DATE

21-01-2022 / 27-01-2022

CITATION

Kikuchi, Kazuro (2022): Theory of noise in optical transmission systems repeated by two-mode phasesensitive optical parametric amplifiers. TechRxiv. Preprint. https://doi.org/10.36227/techrxiv.18841817.v1

$\mathrm{DOI}$

10.36227/techrxiv.18841817.v1 


\title{
Theory of noise in optical transmission systems repeated by two-mode phase-sensitive optical parametric amplifiers
}

\author{
Kazuro Kikuchi \\ National Institution for Academic Degrees and \\ Quality Enhancement of Higher Education
}

\begin{abstract}
We analyze the noise characteristics of an optical transmission system repeated by two-mode phase-sensitive optical parametric amplifiers. In such a system, the non-degenerated signal and idler waves co-propagate through the link, interacting with each other in the optical parametric amplifiers. For the first time, we derive a closed-form expression for the accumulated noise using the quantum-mechanical Langevin-equation approach. The derived noise formula allows calculating the noise figure of the optical-amplifier chain under the influence of the internal loss of the amplifiers and the transmission loss of the link, thereby theoretically demonstrating its low-noise characteristics.
\end{abstract}

\section{Introduction}

Optical parametric amplifiers (OPAs) are suitable for large-capacity long-dis- tance optical transmission systems because of their low noise and wideband characteristics. Recently, phase-insensitive OPAs (PIAs) operating over a 10-THz bandwidth were experimentally demonstrated using periodically poled lithium niobate (PPLN) waveguides. The PIA-based repeaters enabled the transmission of a 41-ch 800-Gb/s polarization-division-multiplexed signal over three $30.8-\mathrm{km}$ repeater spans [1].

However, the noise figure (NF) of the PIA is higher than the quantum limit of $3 \mathrm{~dB}$ because the input port for the idler wave in the PIA is empty, and the vacuum fluctuations in the idler channel merge into the signal. In contrast, one-mode phase-sensitive optical parametric amplifiers (PSAs), in which the signal and idler are degenerated, can perform noise-free optical amplification, that is, NF $=0 \mathrm{~dB}$. This is because there is no empty input port in one-mode PSAs. However, the drawback of one-mode PSAs is that only the in-phase(I) component of the signal electric field is amplified, whereas the quadrature (Q) component is deamplified [2]. Using PPLN waveguides, the in-line one-mode PSA for a 40-Gb/s differential phase-shift-keying signal was demonstrated in a 160-km fiber link [3].

Two-mode PSAs were proposed in [4] and experimentally demonstrated in $[5,6,7]$. They have the advantage that the IQ components of the signal electric field can be simultaneously amplified, while the NF is maintained at $0 \mathrm{~dB}$. Recently, their low noise characteristics were demonstrated by using integrated silicon nitride waveguides [8]. In the transmission system repeated by two-mode PSAs, the idler input is given as a phase conjugation of the signal input. Subsequently, the signal and idler waves co-propagate through the link, interacting with each other in the PSAs. Although it is crucial to investigate the noise characteristics of such a system, there are few studies on this problem.

The present study analyzes the noise characteristics of a transmission system repeated by two-mode PSAs. We apply the quantum-mechanical Langevin-equation approach $[9,10]$ to derive a closed-form expression for the accumulated noise. To the best of the author's knowledge, this is the first study that derives such a theoretical expression. Using the derived noise formula, the NF of the transmission 
system repeated by two-mode PSAs is analyzed under the influence of the internal loss of the PSAs and the transmission loss of the link, demonstrating its low-noise characteristics and elucidating the physical origin of this low noise.

The organization of this paper is as follows. In Sec. 2, following the formulation provided in [9], we introduce the quantum-mechanical Langevin equations for OPAs including the internal loss, and summarize the noise characteristics of PIAs and one-mode PSAs. In Sec. 3, the noise characteristics of an optical-amplifier chain repeated by two-mode PSAs are discussed on the basis of the quantummechanical Langevin-equation approach, and a closed-form expression for the accumulated noise is derived. Section 4 reports calculated results of the NF of single-stage two-mode PSAs. Subsequently, the NF of the optical-amplifier chain repeated by two-mode PSAs is calculated in Sec. 5. Finally, Sec. 6 concludes this paper.

\section{Quantum-mechanical Langevin-equation approach for noise analyses of OPAs}

We introduce the quantum-mechanical Langevin equations for OPAs including the effect of the internal loss. The noise characteristics of PIAs and one-mode PSAs are discussed on the basis of the solutions to the Langevin equations [10].

\subsection{Langevin equations for PIAs and their solutions}

When a signal electric field and an idler electric field propagate through an OPA, the annihilation operator for the signal, $\hat{a}_{s}$, and the creation operator for the idler, $\hat{a}_{i}^{\dagger}$, satisfy the following Langevin equations [11]:

$$
\begin{aligned}
\frac{d \hat{a}_{s}(t)}{d t} & =-\frac{g}{2} e^{i \phi} \hat{a}_{i}^{\dagger}(t)-\frac{\gamma}{2} \hat{a}_{s}(t)+\hat{f}_{s}(t), \\
\frac{d \hat{a}_{i}^{\dagger}(t)}{d t} & =-\frac{g}{2} e^{-i \phi} \hat{a}_{s}(t)-\frac{\gamma}{2} \hat{a}_{i}^{\dagger}(t)+\hat{f}_{i}^{\dagger}(t) .
\end{aligned}
$$

In these equations, the gain coefficient, $g$, is a positive constant determined from the nonlinear optical coefficient and the pump power, and $\phi$ is determined from the phase relation among the signal, idler, and pump waves. In this study, we assume that the loss coefficients of the signal and idler have the same value $\gamma$. The fluctuation operators, $\hat{f}_{s}$ and $\hat{f}_{i}$, result from the loss of the signal and idler, respectively. According to the quantum damping theory [12], the correlation properties between the above-mentioned fluctuation operators are expressed as follows:

$$
\begin{aligned}
\left\langle\hat{f}_{s}(t) \hat{f}_{s}^{\dagger}\left(t^{\prime}\right)\right\rangle & =\gamma \delta\left(t-t^{\prime}\right), \\
\left\langle\hat{f}_{i}(t) \hat{f}_{i}^{\dagger}\left(t^{\prime}\right)\right\rangle & =\gamma \delta\left(t-t^{\prime}\right), \\
\left\langle\hat{f}_{s}^{\dagger}(t) \hat{f}_{s}\left(t^{\prime}\right)\right\rangle & =0, \\
\left\langle\hat{f}_{i}^{\dagger}(t) \hat{f}_{i}\left(t^{\prime}\right)\right\rangle & =0 .
\end{aligned}
$$

Moreover, $\hat{f}_{s}$ and $\hat{f}_{i}$ are not correlated. 
Analytical solutions to Eqs. (1) and (2) can be derived as follows [9, 10]:

$$
\begin{aligned}
\hat{a}_{s}(t) & =\left[\hat{a}_{s}(0)+\hat{A}(t)\right] \cosh \left(\frac{g t}{2}\right) \exp \left(-\frac{\gamma t}{2}\right) \\
& -\left[e^{i \phi} \hat{a}_{i}^{\dagger}(0)+\hat{B}(t)\right] \sinh \left(\frac{g t}{2}\right) \exp \left(-\frac{\gamma t}{2}\right), \\
\hat{a}_{i}^{\dagger}(t) & =\left[\hat{a}_{i}^{\dagger}(0)+e^{-i \phi} \hat{B}(t)\right] \cosh \left(\frac{g t}{2}\right) \exp \left(-\frac{\gamma t}{2}\right) \\
& -e^{-i \phi}\left[\hat{a}_{s}(0)+\hat{A}(t)\right] \sinh \left(\frac{g t}{2}\right) \exp \left(-\frac{\gamma t}{2}\right),
\end{aligned}
$$

where the operators, $\hat{A}(t)$ and $\hat{B}(t)$, are expressed as follows:

$$
\begin{aligned}
& \hat{A}(t)=\int_{0}^{t}\left[\hat{f}_{s}\left(t^{\prime}\right) \exp \left(\frac{\gamma t^{\prime}}{2}\right) \cosh \left(\frac{g t^{\prime}}{2}\right)+\hat{f}_{i}^{\dagger}\left(t^{\prime}\right) e^{i \phi} \exp \left(\frac{\gamma t^{\prime}}{2}\right) \sinh \left(\frac{g t^{\prime}}{2}\right)\right] d t^{\prime}, \\
& \hat{B}(t)=\int_{0}^{t}\left[\hat{f}_{i}^{\dagger}\left(t^{\prime}\right) e^{i \phi} \exp \left(\frac{\gamma t^{\prime}}{2}\right) \cosh \left(\frac{g t^{\prime}}{2}\right)+\hat{f}_{s}\left(t^{\prime}\right) \exp \left(\frac{\gamma t^{\prime}}{2}\right) \sinh \left(\frac{g t^{\prime}}{2}\right)\right] d t^{\prime} .
\end{aligned}
$$

Using Eqs. (3)-(6), we obtain the following averages for the operators, $\hat{A}(t)$ and $\hat{B}(t)$, from Eqs. (9) and (10):

$$
\begin{aligned}
& \left\langle\hat{A}^{\dagger}(t) \hat{A}(t)\right\rangle \\
& =\frac{1}{4}\left[\frac{\exp ((g+\gamma) t)-1}{1+\frac{g}{\gamma}}+\frac{\exp ((-g+\gamma) t)-1}{1-\frac{g}{\gamma}}-2(\exp (\gamma t)-1)\right], \\
& \left\langle\hat{B}^{\dagger}(t) \hat{B}(t)\right\rangle \\
& =\frac{1}{4}\left[\frac{\exp ((g+\gamma) t)-1}{1+\frac{g}{\gamma}}+\frac{\exp ((-g+\gamma) t)-1}{1-\frac{g}{\gamma}}+2(\exp (\gamma t)-1)\right], \\
& \left\langle\hat{A}^{\dagger}(t) \hat{B}(t)+\hat{B}^{\dagger}(t) \hat{A}(t)\right\rangle \\
& =\frac{1}{2}\left[\frac{\exp ((g+\gamma) t)-1}{1+\frac{g}{\gamma}}-\frac{\exp ((-g+\gamma) t)-1}{1-\frac{g}{\gamma}}\right] .
\end{aligned}
$$

When the signal is incident on the OPA but the idler is not, the signal gain does not depend on $\phi$, as shown by Eq. (7). This mode of operation is referred to as phase-insensitive parametric amplification. In the following, we assume that $\hat{a}_{i}(0)=\Delta \hat{a}_{i}(0)$, where $\Delta \hat{a}_{i}(0)$ represents the vacuum fluctuations in the idler channel. According to Eq. (7), the vacuum fluctuations in the idler channel merge into the signal channel and increase the signal noise.

Using Eqs. (7) and (11)-(13), the average number of output signal photons can be derived after straightforward calculations as follows $[9,10]$ :

$$
\left\langle\hat{a}_{s}^{\dagger}(t) \hat{a}_{s}(t)\right\rangle=G_{0} \Gamma_{0}\left\langle\hat{a}_{s}^{\dagger}(0) \hat{a}_{s}(0)\right\rangle+\left(G_{0} \Gamma_{0}-1\right) n_{s p 0},
$$

where the gross gain, $G_{0}$, is given by

$$
G_{0}=\cosh ^{2}\left(\frac{g t}{2}\right),
$$


the internal loss, $\Gamma_{0}$, is given by

$$
\Gamma_{0}=\exp (-\gamma t),
$$

and the spontaneous emission factor, $n_{s p 0}$, is expressed as follows:

$$
\begin{aligned}
n_{s p 0} & =1+\frac{1}{G_{0} \Gamma_{0}-1}\left\{\frac{1}{4\left(1+\frac{g}{\gamma}\right)}\left[1-\Gamma_{0}\left(\sqrt{G_{0}}-\sqrt{G_{0}-1}\right)^{2}\right]\right. \\
& \left.+\frac{1}{4\left(1-\frac{g}{\gamma}\right)}\left[1-\Gamma_{0}\left(\sqrt{G_{0}}+\sqrt{G_{0}-1}\right)^{2}\right]+\frac{1}{2}\left(1-\Gamma_{0}\right)\right\} .
\end{aligned}
$$

The first term in Eq. (14) represents the amplified number of photons, whereas the second term represents the amplified spontaneous emission (ASE), characterized by its resemblance with the laseramplifier theory [13].

From Eq. (7), we find that the real part of the electric field, $\hat{a}_{s r}(t)$, is amplified such that

$$
\left\langle\hat{a}_{s r}(t)\right\rangle=\sqrt{G_{0} \Gamma_{0}}\left\langle\hat{a}_{s r}(0)\right\rangle .
$$

Meanwhile, when the input signal is in the coherent state, the variance of the fluctuations in the real part of the signal electric field is calculated using Eqs. (7), (11)-(13), and (15)-(17), as follows [9]:

$$
\left\langle\Delta \hat{a}_{s r}^{2}(t)\right\rangle=\frac{1}{4}+\frac{1}{2}\left(G_{0} \Gamma_{0}-1\right) n_{s p 0} .
$$

The first term in Eq. (19) represents the vacuum fluctuations, whereas the second term represents the ASE. In addition, the imaginary part has gain and noise variance, which are expressed as in Eqs. (18) and (19), respectively, meaning that PIAs isotropically amplify the IQ components of the signal electric field.

In the optical transmission system, where PIA-based repeaters compensate for the span loss, $\Gamma_{s}$, the net gain of each PIA is adjusted such that

$$
G_{0} \Gamma_{0}=\frac{1}{\Gamma_{s}} .
$$

The ASE from each PIA is accumulated at the output, and the variance of the fluctuations in the real part of the signal electric field is expressed as follows:

$$
\left\langle\Delta \hat{a}_{s r, n}^{2}\right\rangle=\frac{1}{4}+\frac{1}{2}\left(1-\Gamma_{s}\right) n n_{s p 0},
$$

where $n$ represents the number of spans.

\subsection{Langevin equations for one-mode PSAs and their solutions}

When the signal and idler OPAs are degenerated, the gain becomes dependent on $\phi$. This mode of operation is referred to as one-mode phase-sensitive parametric amplification. In contrast to PIAs, the vacuum fluctuations cannot be merged from the idler port, which is closed in one-mode PSAs; therefore, noise-free amplification is possible using such amplifiers.

Given that $\phi=\pi$, Eqs. (1) and (2) yield the following Langevin equation governing the one-mode PSA:

$$
\frac{d \hat{a}_{s}(t)}{d t}=\frac{g}{2} \hat{a}_{s}^{\dagger}(t)-\frac{\gamma}{2} \hat{a}_{s}(t)+\hat{f}_{s}(t) .
$$


The Langevin equations for the real part, $\hat{a}_{s r}(t)$, and imaginary part, $\hat{a}_{s i}(t)$, of the signal electric field are expressed as follows:

$$
\begin{aligned}
\frac{d \hat{a}_{s r}(t)}{d t} & =\frac{g}{2} \hat{a}_{s r}(t)-\frac{\gamma}{2} \hat{a}_{s r}(t)+\frac{1}{2}\left(\hat{f}_{s}(t)+\hat{f}_{s}^{\dagger}(t)\right), \\
\frac{d \hat{a}_{s i}(t)}{d t} & =-\frac{g}{2} \hat{a}_{s i}(t)-\frac{\gamma}{2} \hat{a}_{s i}(t)+\frac{1}{2 i}\left(\hat{f}_{s}(t)-\hat{f}_{s}^{\dagger}(t)\right) .
\end{aligned}
$$

The solution to Eq. (23) is given by

$$
\hat{a}_{s r}(t)=\exp \left(\frac{(g-\gamma) t}{2}\right)\left[\hat{a}_{s r}(0)+\frac{1}{2} \int_{0}^{t} \exp \left(-\frac{(g-\gamma) t^{\prime}}{2}\right)\left(\hat{f}_{s}\left(t^{\prime}\right)+\hat{f}_{s}^{\dagger}\left(t^{\prime}\right)\right) d t^{\prime}\right],
$$

whereas the solution to Eq. (24) is given by

$$
\hat{a}_{s i}(t)=\exp \left(-\frac{(g+\gamma) t}{2}\right)\left[\hat{a}_{s i}(0)+\frac{1}{2 i} \int_{0}^{t} \exp \left(\frac{(g+\gamma) t^{\prime}}{2}\right)\left(\hat{f}_{s}\left(t^{\prime}\right)-\hat{f}_{s}^{\dagger}\left(t^{\prime}\right)\right) d t^{\prime}\right] .
$$

Note that the real part of the signal electric field is amplified, whereas its imaginary part is deamplified, as expressed by

$$
\begin{aligned}
& \left\langle\hat{a}_{s r}(t)\right\rangle=\sqrt{G_{0+} \Gamma_{0}}\left\langle\hat{a}_{s r}(0)\right\rangle, \\
& \left\langle\hat{a}_{s i}(t)\right\rangle=\sqrt{G_{0-} \Gamma_{0}}\left\langle\hat{a}_{s i}(0)\right\rangle,
\end{aligned}
$$

where the phase-sensitive gains, $G_{0+}$ and $G_{0-}$, are defined as follows:

$$
\begin{aligned}
& G_{0+}=\sqrt{G_{0}}+\sqrt{G_{0}-1}, \\
& G_{0-}=\sqrt{G_{0}}-\sqrt{G_{0}-1} .
\end{aligned}
$$

The variance of the fluctuations in the real part of the signal electric field can be calculated from Eqs. (3)-(6) and (25) when the incident signal is in a coherent state, as follows [9, 10]:

$$
\left\langle\Delta \hat{a}_{s r}^{2}(t)\right\rangle=\frac{1}{4}+\frac{\left(G_{0+} \Gamma_{0}-1\right) n_{s p+}}{4},
$$

where $n_{s p+}$ represents the spontaneous emission factor, which is expressed as

$$
n_{s p+}=\frac{g}{g-\gamma} .
$$

Meanwhile, the variance of the imaginary part is calculated from Eqs. (3)-(6) and (26), as follows [10]:

$$
\left\langle\Delta \hat{a}_{s i}^{2}(t)\right\rangle=\frac{1}{4}+\frac{1}{4}\left(G_{0-} \Gamma_{0}-1\right) n_{s p-},
$$

where the spontaneous emission factor, $n_{s p-}$, is defined as

$$
n_{s p-}=\frac{g}{g+\gamma} .
$$

The real part of the signal electric field can be transmitted over a long distance by using one-mode PSA-based repeaters, whose net phase-sensitive gain, $G_{0+} \Gamma_{0}$, is adjusted such that

$$
G_{0+} \Gamma_{0}=\frac{1}{\Gamma_{s}} .
$$

Consequently, the variance of the fluctuations in the real part of the signal electric field is given by

$$
\left\langle\Delta \hat{a}_{s r, n}^{2}\right\rangle=\frac{1}{4}+\frac{1}{4}\left(1-\Gamma_{s}\right) n n_{s p+} .
$$




\section{Analyses of noise in optical transmission systems repeated by two-mode PSAs}

The noise characteristics of an optical-amplifier chain repeated by two-mode PSAs are discussed using the quantum-mechanical Langevin-equation approach introduced in Sec. 2. We derive a closed-form expression for the accumulated noise in the transmitted signal.

\subsection{Langevin equations for two-mode PSAs and their solutions}

Figure 1 illustrates the model for the optical transmission system repeated by two-mode PSAs, where the signal and idler simultaneously travel through the link. Let the annihilation operator for a signal electric field generated from the $k$-th span be $\hat{a}_{s, k}$, and the creation operator for an idler electric field generated from the $k$-th span be $\hat{a}_{i, k}^{\dagger}$. When the phase relation among the signal, idler, and pump is optimally controlled in each PSA such that $\phi=\pi$, Eqs. (7) and (8) yield the following difference sequences for $\hat{a}_{s, k}$ and $\hat{a}_{i, k}^{\dagger}$ :

$$
\begin{aligned}
& \hat{a}_{s, k+1}=\left(\sqrt{G_{0} \Gamma_{0}} \hat{a}_{s, k}+\sqrt{\left(G_{0}-1\right) \Gamma_{0}} \hat{a}_{i, k}^{\dagger}+\hat{C}_{s, k}^{\dagger}+\hat{D}_{s, k}\right) \sqrt{\Gamma_{s}}+\sqrt{1-\Gamma_{s}} \hat{d}_{s, k} \\
& \hat{a}_{i, k+1}^{\dagger}=\left(\sqrt{G_{0} \Gamma_{0}} \hat{a}_{i, k}^{\dagger}+\sqrt{\left(G_{0}-1\right) \Gamma_{0}} \hat{a}_{s, k}+\hat{C}_{i, k}^{\dagger}+\hat{D}_{i, k}\right) \sqrt{\Gamma_{s}}+\sqrt{1-\Gamma_{s}} \hat{d}_{i, k}^{\dagger} .
\end{aligned}
$$

The operators, $\hat{C}_{s, k}^{\dagger}, \hat{D}_{s, k}, \hat{C}_{i, k}^{\dagger}$, and $\hat{D}_{i, k}$, represent fluctuations that originate from the internal loss of the signal and idler, and are expressed as follows:

$$
\begin{aligned}
& \hat{C}_{s, k}^{\dagger}=\int_{0}^{t} \hat{f}_{i, k}^{\dagger}\left(t^{\prime}\right) \exp \left(\frac{\gamma t^{\prime}}{2}\right)\left[\sqrt{\left(G_{0}-1\right) \Gamma_{0}} \cosh \left(\frac{g t^{\prime}}{2}\right)-\sqrt{G_{0} \Gamma_{0}} \sinh \left(\frac{g t^{\prime}}{2}\right)\right] d t^{\prime}, \\
& \hat{D}_{s, k}=\int_{0}^{t} \hat{f}_{s, k}\left(t^{\prime}\right) \exp \left(\frac{\gamma t^{\prime}}{2}\right)\left[\sqrt{G_{0} \Gamma_{0}} \cosh \left(\frac{g t^{\prime}}{2}\right)-\sqrt{\left(G_{0}-1\right) \Gamma_{0}} \sinh \left(\frac{g t^{\prime}}{2}\right)\right] d t^{\prime}, \\
& \hat{C}_{i, k}^{\dagger}=\int_{0}^{t} \hat{f}_{i, k}^{\dagger}\left(t^{\prime}\right) \exp \left(\frac{\gamma t^{\prime}}{2}\right)\left[\sqrt{G_{0} \Gamma_{0}} \cosh \left(\frac{g t^{\prime}}{2}\right)-\sqrt{\left(G_{0}-1\right) \Gamma_{0}} \sinh \left(\frac{g t^{\prime}}{2}\right)\right] d t^{\prime}, \\
& \hat{D}_{i, k}=\int_{0}^{t} \hat{f}_{s, k}\left(t^{\prime}\right) \exp \left(\frac{\gamma t^{\prime}}{2}\right)\left[\sqrt{\left(G_{0}-1\right) \Gamma_{0}} \cosh \left(\frac{g t^{\prime}}{2}\right)-\sqrt{G_{0} \Gamma_{0}} \sinh \left(\frac{g t^{\prime}}{2}\right)\right] d t^{\prime} .
\end{aligned}
$$

The fluctuation operators, $\hat{f}_{s, k}$ and $\hat{f}_{i, k}$, result from the internal loss of the signal and idler in the $k$-th PSA, respectively, and they satisfy the correlation relations expressed by Eqs. (3)-(6). Moreover, in Eqs. (37) and (38), the annihilation operator for the vacuum field associated with the span loss of the signal is given by $\hat{d}_{s, k}$, and the creation operator for the vacuum field associated with the span loss of the idler is given by $\hat{d}_{i, k}^{\dagger}$.

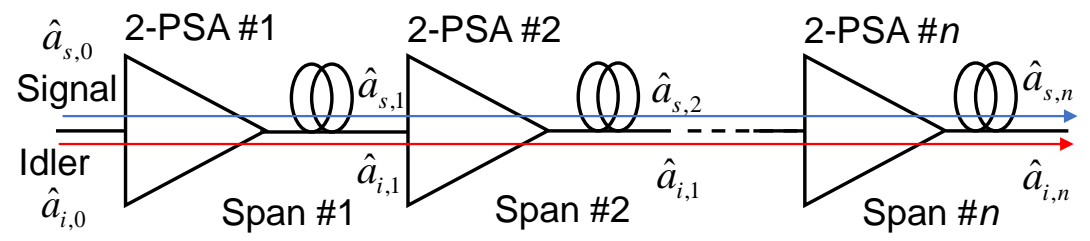

Figure 1: Transmission system repeated by two-mode PSAs; the signal and idler simultaneously travel through the link. 
Equations (37) and (38) are transformed into

$$
\begin{aligned}
\hat{a}_{s, k+1}+\hat{a}_{i, k+1}^{\dagger} & =\sqrt{G_{+}}\left(\hat{a}_{s, k}+\hat{a}_{i, k}^{\dagger}\right)+\sqrt{\Gamma_{s}}\left(\hat{C}_{s, k}^{\dagger}+\hat{C}_{i, k}^{\dagger}+\hat{D}_{s, k}+\hat{D}_{i, k}\right) \\
& +\sqrt{1-\Gamma_{s}}\left(\hat{d}_{s, k}+\hat{d}_{i, k}^{\dagger}\right), \\
\hat{a}_{s, k+1}-\hat{a}_{i, k+1}^{\dagger} & =\sqrt{G_{-}}\left(\hat{a}_{s, k}-\hat{a}_{i, k}^{\dagger}\right)+\sqrt{\Gamma_{s}}\left(\hat{C}_{s, k}^{\dagger}-\hat{C}_{i, k}^{\dagger}+\hat{D}_{s, k}-\hat{D}_{i, k}\right) \\
& +\sqrt{1-\Gamma_{s}}\left(\hat{d}_{s, k}-\hat{d}_{i, k}^{\dagger}\right),
\end{aligned}
$$

where the net phase-sensitive gains, $G_{ \pm}$, are defined as

$$
G_{ \pm}=G_{0 \pm} \Gamma_{0} \Gamma_{s}
$$

We find that $\hat{a}_{s, 0}+\hat{a}_{i, 0}^{\dagger}$ is amplified with a gain $\sqrt{G_{+}}$in each span, whereas $\hat{a}_{s, 0}-\hat{a}_{i, 0}^{\dagger}$ is deamplified with a factor $\sqrt{G_{-}}$. Consequently, the solutions to Eqs. (43) and (44) are expressed as follows:

$$
\begin{aligned}
\hat{a}_{s, n}+\hat{a}_{i, n}^{\dagger} & =\sqrt{G_{+}^{n}}\left(\hat{a}_{s, 0}+\hat{a}_{i, 0}^{\dagger}\right)+\sqrt{\Gamma_{s}} \sum_{k=1}^{n} \sqrt{G_{+}^{k-1}}\left(\hat{C}_{s, k}^{\dagger}+\hat{D}_{s, k}+\hat{C}_{i, k}^{\dagger}+\hat{D}_{i, k}\right) \\
& +\sqrt{1-\Gamma_{s}} \sum_{k=1}^{n} \sqrt{G_{+}^{k-1}}\left(\hat{d}_{s, k}+\hat{d}_{i, k}^{\dagger}\right) \\
\hat{a}_{s, n}-\hat{a}_{i, n}^{\dagger} & =\sqrt{G_{-}^{n}}\left(\hat{a}_{s, 0}-\hat{a}_{i, 0}^{\dagger}\right)+\sqrt{\Gamma_{s}} \sum_{k=1}^{n} \sqrt{G_{-}^{k-1}}\left(\hat{C}_{s, k}^{\dagger}+\hat{D}_{s, k}-\hat{C}_{i, k}^{\dagger}-\hat{D}_{i, k}\right) \\
& +\sqrt{1-\Gamma_{s}} \sum_{k=1}^{n} \sqrt{G_{-}^{k-1}}\left(\hat{d}_{s, k}-\hat{d}_{i, k}^{\dagger}\right) .
\end{aligned}
$$

Finally, from Eqs. (46) and (47), we obtain the following expression for the signal output from the $n$-th span:

$$
\begin{aligned}
\hat{a}_{s, n} & =\frac{1}{2}\left[\left(\sqrt{G_{+}^{n}}+\sqrt{G_{-}^{n}}\right) \hat{a}_{s, 0}+\left(\sqrt{G_{+}^{n}}-\sqrt{G_{-}^{n}}\right) \hat{a}_{i, 0}^{\dagger}\right] \\
& +\frac{\sqrt{\Gamma_{s}}}{2}\left[\sum_{k=1}^{n}\left(\sqrt{G_{+}^{k-1}}+\sqrt{G_{-}^{k-1}}\right)\left(\hat{C}_{s, k}^{\dagger}+\hat{D}_{s, k}\right)+\sum_{k=1}^{n}\left(\sqrt{G_{+}^{k-1}}-\sqrt{G_{-}^{k-1}}\right)\left(\hat{C}_{i, k}^{\dagger}+\hat{D}_{i, k}\right)\right] \\
& +\frac{\sqrt{1-\Gamma_{s}}}{2}\left[\sum_{k=1}^{n}\left(\sqrt{G_{+}^{k-1}}+\sqrt{G_{-}^{k-1}}\right) \hat{d}_{s, k}+\sum_{k=1}^{n}\left(\sqrt{G_{+}^{k-1}}-\sqrt{G_{-}^{k-1}}\right) \hat{d}_{i, k}^{\dagger}\right] .
\end{aligned}
$$

Preparing the idler input such that $\left\langle\hat{a}_{i, 0}\right\rangle=\left\langle\hat{a}_{s, 0}\right\rangle^{*}$ through four-wave mixing and proper phase adjustment processes [5], we can obtain the following expression for signal output:

$$
\left\langle\hat{a}_{s, n}\right\rangle=\sqrt{G_{+}^{n}}\left\langle\hat{a}_{s, 0}\right\rangle .
$$

Therefore, to compensate for the span loss, the gain of each amplifier is adjusted such that $G_{+}=1$. Note that both IQ components of the signal electric field can be transmitted even exploiting the phase-sensitive gain. 


\subsection{Close-form expression for noise in two-mode PSAs}

Let the real parts of $\hat{C}_{s, k}, \hat{C}_{i, k}, \hat{D}_{s, k}$, and $\hat{D}_{i, k}$ be expressed as $\hat{C}_{s r, k}, \hat{C}_{i r, k}, \hat{D}_{s r, k}$, and $\hat{D}_{i r, k}$, respectively. Equations (39)-(42) yield the following averages for the operators:

$$
\begin{aligned}
\left\langle\hat{C}_{s r, k}^{2}\right\rangle & =\left\langle\hat{D}_{i r, k}^{2}\right\rangle=\frac{1}{4}\left[\frac{\gamma}{4(g+\gamma)}\left(1-\Gamma_{0} G_{0-}\right)+\frac{\gamma}{4(-g+\gamma)}\left(1-\Gamma_{0} G_{0+}\right)-\frac{1-\Gamma_{0}}{2}\right], \\
\left\langle\hat{C}_{i r, k}^{2}\right\rangle & =\left\langle\hat{D}_{s r, k}^{2}\right\rangle=\frac{1}{4}\left[\frac{\gamma}{4(g+\gamma)}\left(1-\Gamma_{0} G_{0-}\right)+\frac{\gamma}{4(-g+\gamma)}\left(1-\Gamma_{0} G_{0+}\right)+\frac{1-\Gamma_{0}}{2}\right], \\
\left\langle\hat{C}_{s r, k} \hat{C}_{i r, k}\right\rangle & =\left\langle\hat{D}_{s r, k} \hat{D}_{i r, k}\right\rangle=\frac{1}{16}\left[\frac{\gamma}{-g+\gamma}\left(1-\Gamma_{0} G_{0+}\right)-\frac{\gamma}{g+\gamma}\left(1-\Gamma_{0} G_{0-}\right)\right] .
\end{aligned}
$$

Using Eqs. (50)-(52), Eq. (48) yields the variance of the fluctuations in the real part of the signal electric field from the $n$-th span, which is expressed as follows:

$$
\begin{aligned}
\left\langle\Delta \hat{a}_{s r, n}^{2}\right\rangle & =\frac{1}{8}\left(G_{+}^{n}+G_{-}^{n}\right)+\frac{\Gamma_{s}}{2}\left[\left(\sum_{k=1}^{n} G_{+}^{k-1}\right) \frac{\gamma}{4(-g+\gamma)}\left(1-\Gamma_{0} G_{0+}\right)\right. \\
& \left.+\left(\sum_{k=1}^{n} G_{-}^{k-1}\right) \frac{\gamma}{4(g+\gamma)}\left(1-\Gamma_{0} G_{0-}\right)\right]+\frac{1-\Gamma_{s}}{8} \sum_{k=1}^{n}\left(G_{+}^{k-1}+G_{-}^{k-1}\right) .
\end{aligned}
$$

\section{NF of single-stage two-mode PSAs}

After deriving the theoretical expression for the NF of single-stage two-mode PSAs, we calculate the $\mathrm{NF}$ as functions of the net gain and internal loss.

\subsection{Theoretical expression for NF}

The average of the real part of the signal electric field from the single-stage two-mode PSA is expressed as follows:

$$
\left\langle\hat{a}_{s r, 1}\right\rangle=\sqrt{G_{+}}\left\langle\hat{a}_{s r, 0}\right\rangle .
$$

Meanwhile, the variance of its fluctuations is derived from Eq. (53), setting $n=1$ and $\Gamma_{s}=1$, as follows:

$$
\left\langle\Delta \hat{a}_{s r, 1}^{2}\right\rangle=\frac{1}{4}+\frac{\left(G_{+}-1\right) n_{s p+}}{8}+\frac{\left(G_{-}-1\right) n_{s p-}}{8},
$$

which demonstrates that the noise of the two-mode PSA stems from the vacuum fluctuations and ASE associated with the phase-sensitive gains of $G_{ \pm}$. The equivalent input noise is expressed as follows:

$$
\left\langle\Delta \hat{a}_{s r, 1, e q}^{2}\right\rangle=\frac{\left\langle\Delta \hat{a}_{s r, 1}^{2}\right\rangle}{G_{+}} .
$$

Meanwhile, fluctuations of the input signal and idler are expressed as follows:

$$
\begin{aligned}
\left\langle\Delta \hat{a}_{s r, 0}^{2}\right\rangle & =\frac{1}{4}, \\
\left\langle\Delta \hat{a}_{i r, 0}^{2}\right\rangle & =\frac{1}{4} .
\end{aligned}
$$

Note that the signal and idler have the same modulation but their noise is uncorrelated. Thus, we can consider that the noise variance of the input signal reduces to $1 / 8$ through the averaging process. The 
NF of amplifiers can be generally defined as the power ratio of the equivalent input noise to the actual input noise. Consequently, the NF of the single-stage two-mode PSA is obtained as follows:

$$
\mathrm{NF}=8\left\langle\Delta \hat{a}_{s r, 1, e q}^{2}\right\rangle .
$$

In the limit where $G_{+} \rightarrow \infty$, Eq. (59) leads to

$$
\mathrm{NF}=n_{s p+},
$$

which is the same as the NF of the one-mode PAS. We find that when $g \gg \gamma$, the NF approaches $0 \mathrm{~dB}$.

\subsection{Numerical results on NF}

Figure 2 represents the NFs of single-stage two-mode PSAs calculated as a function of the net gain, $G_{+}$. The black, red, blue, and green curves correspond to $\Gamma_{0}$ values of $0 \mathrm{~dB},-3 \mathrm{~dB},-5 \mathrm{~dB}$, and $-10 \mathrm{~dB}$, respectively. The solid curves were calculated using Eqs. (55), (56), and (59), whereas the broken curves were obtained from the approximated formula provided in Eq. (60). We find that $n_{s p+}$ is a good approximation of $\mathrm{NF}$ when $G_{+}$is sufficiently larger than unity.

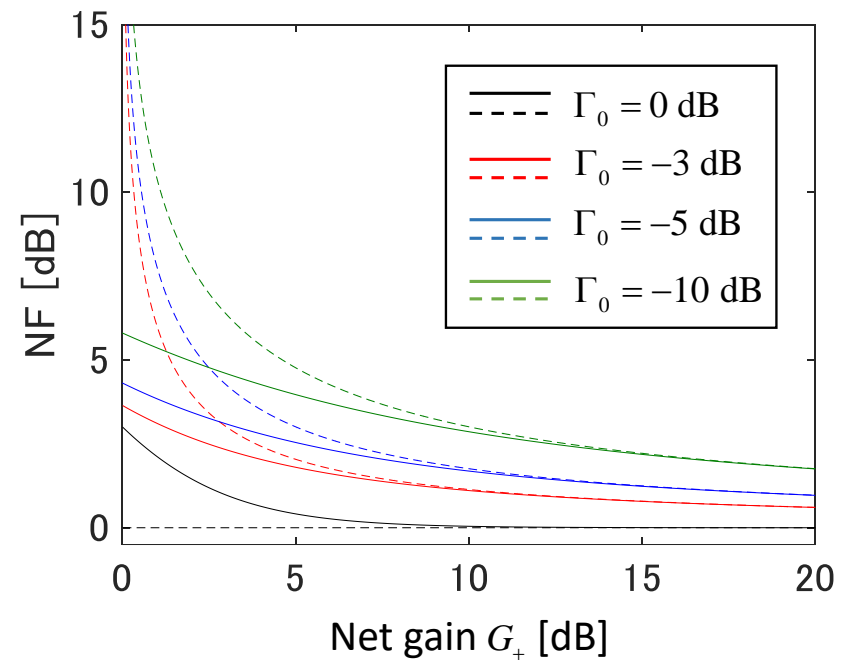

Figure 2: NFs of single-stage two-mode PSAs calculated as a function of the net gain $G_{+}$, when $\Gamma_{0}$ was set to $0 \mathrm{~dB}$ (black), $-3 \mathrm{~dB}$ (red), $-5 \mathrm{~dB}$ (blue), and $-10 \mathrm{~dB}$ (green). The solid curves were calculated using Eqs. (55), (56), and (59), whereas the broken curves were obtained from the approximated formula $\mathrm{NF}=n_{s p+}$.

Figure 3 depicts the NFs of single-stage two-mode PSAs calculated as a function of the internal loss expressed as $1 / \Gamma_{0}$. The blue, red, and black curves represent the results obtained when the net gain $G_{+}$was fixed at $5 \mathrm{~dB}, 10 \mathrm{~dB}$, and $20 \mathrm{~dB}$, respectively. The solid curves were obtained from Eqs. (55), (56), and (59), whereas the broken curves were obtained from the approximated formula provide in Eq. (60). This approximated formula is valid when $G_{+} \gtrsim 10 \mathrm{~dB}$.

\section{$5 \quad$ NF of an optical-amplifier chain using two-mode PSAs}

We next derive the theoretical expression for the NF of the optical-amplifier chain repeated by twomode PSAs and calculate the NF as a function of the number of spans with the preamplifier gain and internal loss as parameters. 


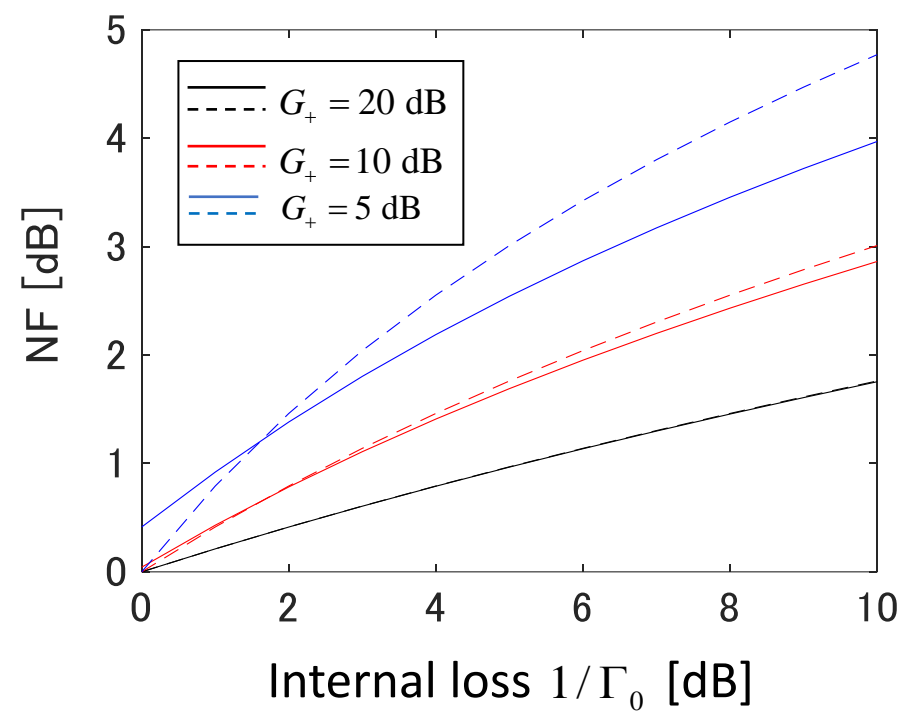

Figure 3: NFs of single-stage two-mode PSAs calculated as a function of the internal loss $1 / \Gamma_{0}$, when $G_{+}$was set to $5 \mathrm{~dB}$ (blue), $10 \mathrm{~dB}$ (red), and $20 \mathrm{~dB}$ (black). The solid curves were calculated using Eqs. (55), (56), and (59), whereas the broken curves were obtained from the approximated formula $\mathrm{NF}=n_{s p+}$.

\subsection{Theoretical expression for NF}

We assume the use of an optical preamplifier, which is an ideal two-mode PSA without internal loss, in an optical transmission system repeated by two-mode PSAs. The phase-sensitive gains of the preamplifier are $G_{+}^{\prime}(\gg 1)$ and $G_{-}^{\prime}\left(=1 / G_{+}^{\prime}\right)$. Consequently, Eq. (53) is modified as follows:

$$
\begin{aligned}
\left\langle\Delta \hat{a}_{s r, n}^{2}\right\rangle & =\frac{1}{8}\left(G_{+}^{n} G_{+}^{\prime}+G_{-}^{n} G_{-}^{\prime}\right)+\frac{\Gamma_{s}}{2}\left[\left(\sum_{k=1}^{n} G_{+}^{k-1}\right) \frac{\gamma}{4(-g+\gamma)}\left(1-\Gamma_{0} G_{0+}\right) G_{+}^{\prime}\right. \\
& \left.+\left(\sum_{k=1}^{n} G_{-}^{k-1}\right) \frac{\gamma}{4(g+\gamma)}\left(1-\Gamma_{0} G_{0-}\right) G_{-}^{\prime}\right] \\
& +\frac{1-\Gamma_{s}}{8}\left[\left(\sum_{k=1}^{n} G_{+}^{k-1}\right) G_{+}^{\prime}+\left(\sum_{k=1}^{n} G_{-}^{k-1}\right) G_{-}^{\prime}\right] .
\end{aligned}
$$

Using Eq. (61), we obtain the equivalent input noise as

$$
\left\langle\Delta \hat{a}_{s r, n, e q}^{2}\right\rangle=\frac{\left\langle\Delta \hat{a}_{s r, n}^{2}\right\rangle}{G_{+}^{\prime}},
$$

and the NF of the optical-amplifier chain using the two-mode PSAs is expressed as

$$
\mathrm{NF}=8\left\langle\Delta \hat{a}_{s r, n, e q}^{2}\right\rangle \text {. }
$$

Equations (61)-(63) show that when $G_{+}^{\prime} \rightarrow \infty$, the NF is reduced to

$$
\mathrm{NF}=1+\left(1-\Gamma_{s}\right) n n_{s p+},
$$

which is the same as the NF of the optical-amplifier chain using one-mode PSAs. We find that the phase-sensitive gains of the preamplifier, $G_{ \pm}^{\prime}$, can filter the noise, and that the noise associated with 
$G_{-}$is eliminated, as expressed in Eq. (61). Therefore, the NF approaches its theoretical limit given by Eq. (64).

\subsection{Numerical results on NF}

Figure 4 represents the NFs of the optical-amplifier chain using two-mode PSAs calculated as a function of the number of spans, $n$, when $\Gamma_{s}=-10 \mathrm{~dB}$ and $\Gamma_{0}=0 \mathrm{~dB}$. The black, red, and blue circles represent the NFs obtained using Eqs. (61), (62), and (63) when the preamplifier gain $G_{+}^{\prime}$ was set to $0 \mathrm{~dB}, 3 \mathrm{~dB}$, and $10 \mathrm{~dB}$, respectively. The broken curve represents the theoretical limit given by Eq. (64). The blue circles are in good agreement with the theoretical limit. This is because when $G_{+}^{\prime} \gtrsim 10 \mathrm{~dB}$, the noise associated with $G_{-}$in Eq. (61) is sufficiently suppressed.

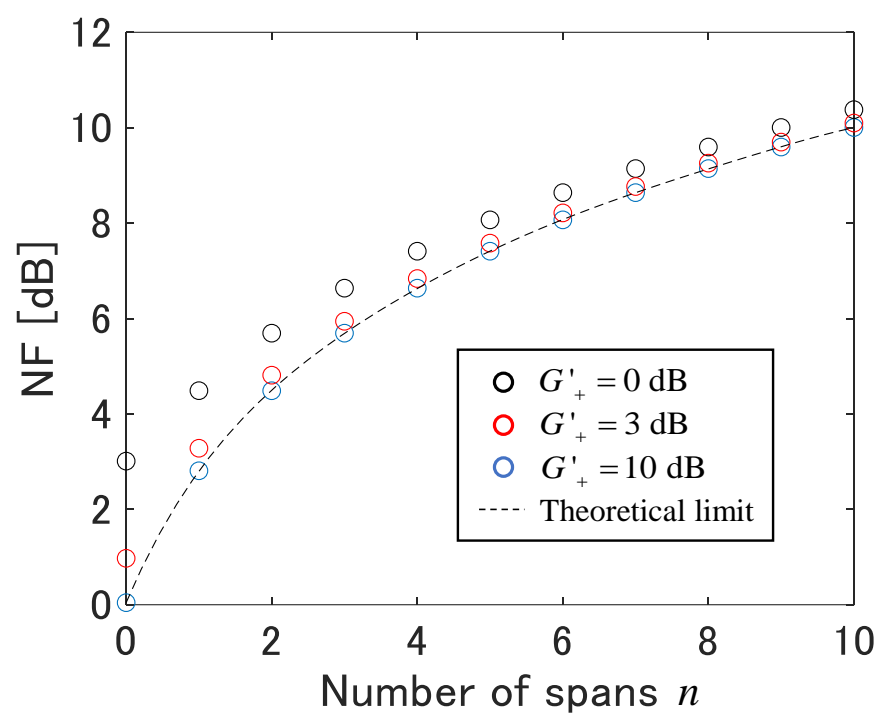

Figure 4: NFs of the optical-amplifier chain using two-mode PSAs calculated as a function of $n$. We assume that $\Gamma_{s}=-10 \mathrm{~dB}$ and $\Gamma_{0}=0 \mathrm{~dB}$. The black, red, and blue circles are the NFs obtained when $G_{+}^{\prime}=0 \mathrm{~dB}, 3 \mathrm{~dB}$, and $10 \mathrm{~dB}$, respectively, using Eqs. (61), (62), and (63). The broken curve shows the theoretical limit given by Eq. (64).

Figure 5 represents NFs calculated as a function of $n$ by using Eqs. (61), (62), and (63), when $G_{+}^{\prime}=10 \mathrm{~dB}$ and $\Gamma_{s}=-10 \mathrm{~dB}$. The black, red, and blue circles correspond to $\Gamma_{0}=0 \mathrm{~dB},-3 \mathrm{~dB}$, and $-10 \mathrm{~dB}$, respectively. In addition, the solid curves are the theoretical limits calculated from Eq. (64). The circles are in good agreement with the theoretical limits owing to the sufficient preamplifier gain, $G_{+}^{\prime}=10 \mathrm{~dB}$.

\section{Conclusions}

We analyzed the noise characteristics of an optical transmission system repeated by two-mode PSAs using the quantum-mechanical Langevin-equation approach. To the best of the author's knowledge, this is the first study that derives a theoretical expression for the amount of accumulated noise. We calculated the NF under the influence of the internal loss of the PSAs and the transmission loss of the link, theoretically demonstrating the low-noise characteristics of the system and elucidating the physical origin of this low noise. 


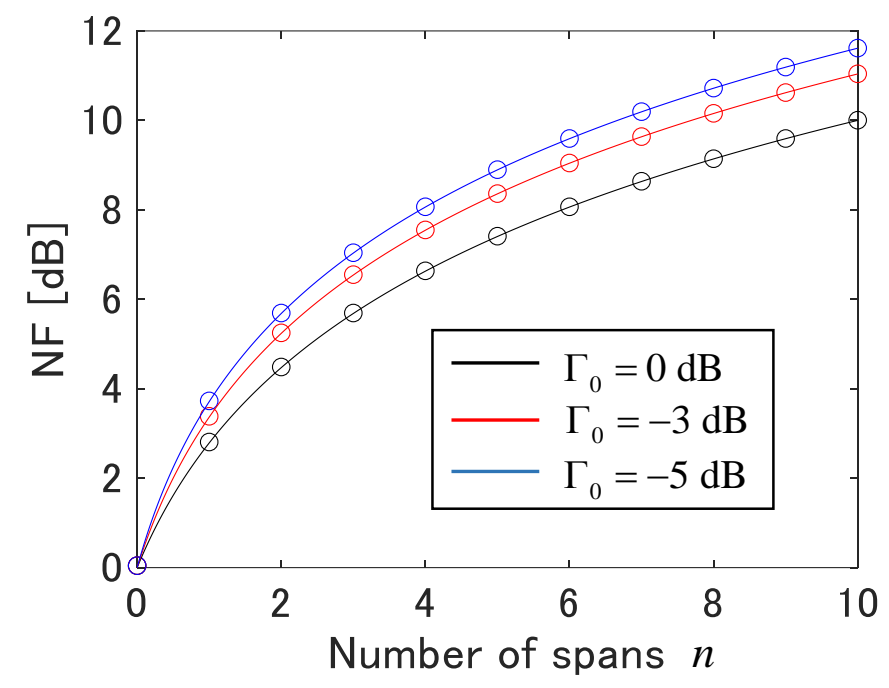

Figure 5: NFs of the optical-amplifier chain using two-mode PSAs calculated as a function of $n$ using Eqs. (61), (62), and (63), when $G_{+}^{\prime}=10 \mathrm{~dB}$ and $\Gamma_{s}=-10 \mathrm{~dB}$. The black, red, and blue circles correspond to $\Gamma_{0}=0 \mathrm{~dB},-3 \mathrm{~dB}$, and $-10 \mathrm{~dB}$, respectively. The solid curves represent the theoretical limits calculated using Eq. (64).

\section{References}

[1] T. Kobayashi, S. Shimizu, M. Nakamura, T. Umeki, T. Kazama, R. Kasahara, F. Hamaoka, M. Nagatani, H. Yamazaki, T. Mizuno, H. Nosaka, and Y. Miyamoto, "Wideband inline-amplified WDM transmission using PPLN-based OPA with over-10-THz bandwidth," in Optical Fiber Communication Conference, OSA Technical Digest (Optical Society of America, 2020), Th4C.7.

[2] K. Kikuchi, "Fundamentals of coherent optical fiber communications," J. Lightwave Technol., vol. 34, no. 1, pp. 157-179, 2016.

[3] T. Umeki, M. Asobe, and H. Takenouchi, "In-line phase sensitive amplifier based on PPLN waveguides," Opt. Express, vol. 21, no. 10, pp. 12077-12084, 2013.

[4] C. J. McKinstrie, N. Alic, Z. Tong, and M. Karlsson, "Higher-capacity communication links based on two-mode phase-sensitive amplifiers," Opt. Express, vol. 19, no. 13, pp. 11977-11991, 2013.

[5] Z. Tong, C. Lundström, P. A. Andrekson, C. J. McKinstrie, M. Karlsson, D. J. Blessing, E. Tipsuwannakul, B. J. Puttnam, H. Toda, and L. Grüner-Nielsen, "Towards ultrasensitive optical links enabled by low-noise phase-sensitive amplifiers," Nature Photonics, vol. 5, pp. 430-436, 2011.

[6] B. Corcoran, S. L. I. Olsson, C. Lundström, M. Karlsson, and P. Andrekson, "Phase-sensitive optical pre-amplifier implemented in an 80km DQPSK link," in Optical Fiber Communication Conference, Postdeadline Papers 2012 (Optical Society of America, 2012), PDP5A.4.

[7] S. L. I. Olsson, H. Eliasson, E. Astra, M. Karlsson, and P. A. Andrekson, "Long-haul optical transmission link using low-noise phase-sensitive amplifiers," Nature Communications, vol. 9, p. 2513, 2018.

[8] P. Zhao, Z. Ye, M. Karlsson, V. Torres-Company, and P. A. Andrekson, "Overcoming the quantum noise limit with continuous-wave phase-sensitive parametric amplification based on a single 
integrated silicon-nitride waveguide," in Optical Fiber Communication Conference, Postdeadline Papers 2021 (Optical Society of America, 2021), F3A.5.

[9] K. Kikuchi, "Theory of spontaneous emission factors of optical parametric amplifiers," in Optical Fiber Communication Conference, OSA Technical Digest (Optical Society of America, 2021), W6A.38.

[10] K. Kikuchi, "Theory of spontaneous emission factor and noise figure of optical parametric amplifiers," TechRxiv Preprint, DOI: 10.36227/techrxiv.18489533.v1, 2022.

[11] A. Yariv, Quantum Electronics (Third Edition). John Wiley \& Sons, Inc., 1989.

[12] M. O. Scully and M. S. Zubairy, Quantum Optics. Cambridge University Press, 1997.

[13] K. Shimoda, H. Takahashi, and C. H. Townes, "Fluctuations in amplification of quanta with application to maser amplifiers," J. Phys. Soc. Jpn., vol. 12, no. 6, pp. 686-700, 1957. 